\title{
Development of a model to predict recurrence after bronchial artery embolization for non-cancer related hemoptysis
}

\author{
Hai-Tao Yan ${ }^{1 \dagger}$, Guang-Dong Lu ${ }^{1 \dagger}$, Xiang-Zhong Huang ${ }^{2}$, Da-Zhong Zhang ${ }^{3}$, Kun-Yuan Ge ${ }^{4}$, Jin-Xing Zhang ${ }^{1}$,
} Jin Liư ${ }^{5}$, Sheng Liu', Hai-Bin Shi ${ }^{1 *}$ and Qing-Quan Zu ${ }^{1 *}$

\begin{abstract}
Background: Relapse after effective bronchial arterial embolization (BAE) for controlling hemoptysis is not uncommon. Studies reported diverse predictors of recurrence. However, a model to assess the probability of recurrence in non-cancer related hemoptysis patients after BAE has not been reported. This study was to develop a model to predict recurrence after BAE for non-cancer related hemoptysis.

Methods: The study cohort included 487 patients who underwent BAE for non-cancer-related hemoptysis between January 2015 and December 2019. We derived the model's variables from univariate and multivariate Cox regression analyses. The model presented as a nomogram scaled by the proportional regression coefficient of each predictor. Model performance was assessed with respect to discrimination and calibration.

Results: One-month and 1-, 2-, 3- and 5-year recurrence-free rates were 94.5\%, 88.0\%, 81.4\%, 76.2\% and 73.8\%, respectively. Risk factors for recurrence were underlying lung diseases and the presence of systemic arterial-pulmonary circulation shunts. This risk prediction model with two risk factors provided good discrimination (area under curve, 0.69; 95\% confidence interval, 0.62-0.76), and lower prediction error (integrated Brier score, 0.143).

Conclusion: The proposed model based on routinely available clinical and imaging features demonstrates good performance for predicting recurrence of non-cancer-related hemoptysis after BAE. The model may assist clinicians in identifying higher-risk patients to improve the long-term efficacy of BAE.
\end{abstract}

Keywords: Bronchial arteries, Embolization, Therapeutic, Hemoptysis, Nomograms, Recurrence

\section{Introduction}

Roughly $70 \%$ of hemoptysis is caused by respiratory diseases unrelated to cancer [1-3]. Bronchial arterial embolization (BAE) is highly effective in controlling hemoptysis. However, recurrence of hemoptysis after

\footnotetext{
*Correspondence: shihb@njmu.edu.cn; zuqingquan@njmu.edu.cn ${ }^{\dagger}$ Hai-Tao Yan and Guang-Dong Lu have contributed equally as first author to this study.

${ }^{1}$ Department of Interventional Radiology, The First Affiliated Hospital With Nanjing Medical University, No. 300 Guangzhou Road, Nanjing 210029, China

Full list of author information is available at the end of the article
}

successful BAE is not uncommon. Extended analysis found that $5-10 \%$ of recurrences occurred within one month after BAE primarily secondary to missed culprit arteries [4]. Long-term recurrence rates fluctuated between 20 and $40 \%$ in patients with noncancer-related hemoptysis $[3,5,6]$. Recanalization, revascularization due to migration of embolized materials, and primary diseases progression remained dominant causes of these recurrent events $[4,7,8]$. Approximately $40-60 \%$ of patients with recurrent hemoptysis had to undergo repeat embolization, lobectomy, or died $[4,9,10]$. Given these disparate outcomes, an 
accurate model to predict recurrent hemoptysis would permit more optimal surveillance, prevention, and management strategies for these patients.

Characteristics, including patient demographics, primary respiratory diseases, thoracic imaging, and embolization procedures, were analyzed for significant prediction values of recurrence [3, 4, 9-13]. BAE showed its superiority of controlling emergency bleeding by embolizing target vessels, not eliminate underlying lung diseases with equivalence effect of curative intent, e.g., lobectomy. Pre-procedure CT imaging and angiographic findings were also assessed for their predictive capability [4]. However, those studies did not fully integrate the clinical and imaging features nor determine their individual contributions to predicting recurrent bleeding. In addition, some researchers combined non-cancer and cancer related hemoptysis in the analysis [14]. In light of the discrepant nature of benign and malignant diseases-associated hemoptysis, a prediction model specific to each group is needed.

In this study, we employed a large, multi-center cohort to develop a risk prediction model for recurrence of non-cancer related hemoptysis after endovascular treatment. The model can be used in evaluating individualized prognosis and in raising precaution for the risk cohort.

\section{Patients and methods}

Ethics committee approval was granted by the local institutional ethics review board for this retrospective study. The requirement for informed consent was waived due to its retrospective nature. All procedures followed in this study conformed to the guidelines of the World Medical Association's Helsinki Declaration (2008). The data that support the findings of this study are available from the corresponding author upon reasonable request.

\section{Patients}

The medical records of 603 patients who underwent arterial embolization for non-cancer-related hemoptysis at four tertiary centers between January 2015 and December 2019 were queried. During hospitalization, all hemoptysis patients underwent the standard care: vital sign monitoring, keeping airway stabilization, correction of hypoxemia, hemostasis, nebulized treatment and empirical antibiotic therapy. The exclusion criteria were: (1) either technique failure (failed attempted at embolization) or clinical failure (failure of hemostasis within $24 \mathrm{~h}$ after embolization) [15]; (2) lack of contrast enhanced thoracic computed tomography (CT); (3) medical record missing; and (4) lack of follow-up data. Based on the criteria, 487 patients (348 men and 139 women) were included in the study cohort (Fig. 1). From the medical records, information deemed related to hemoptysis

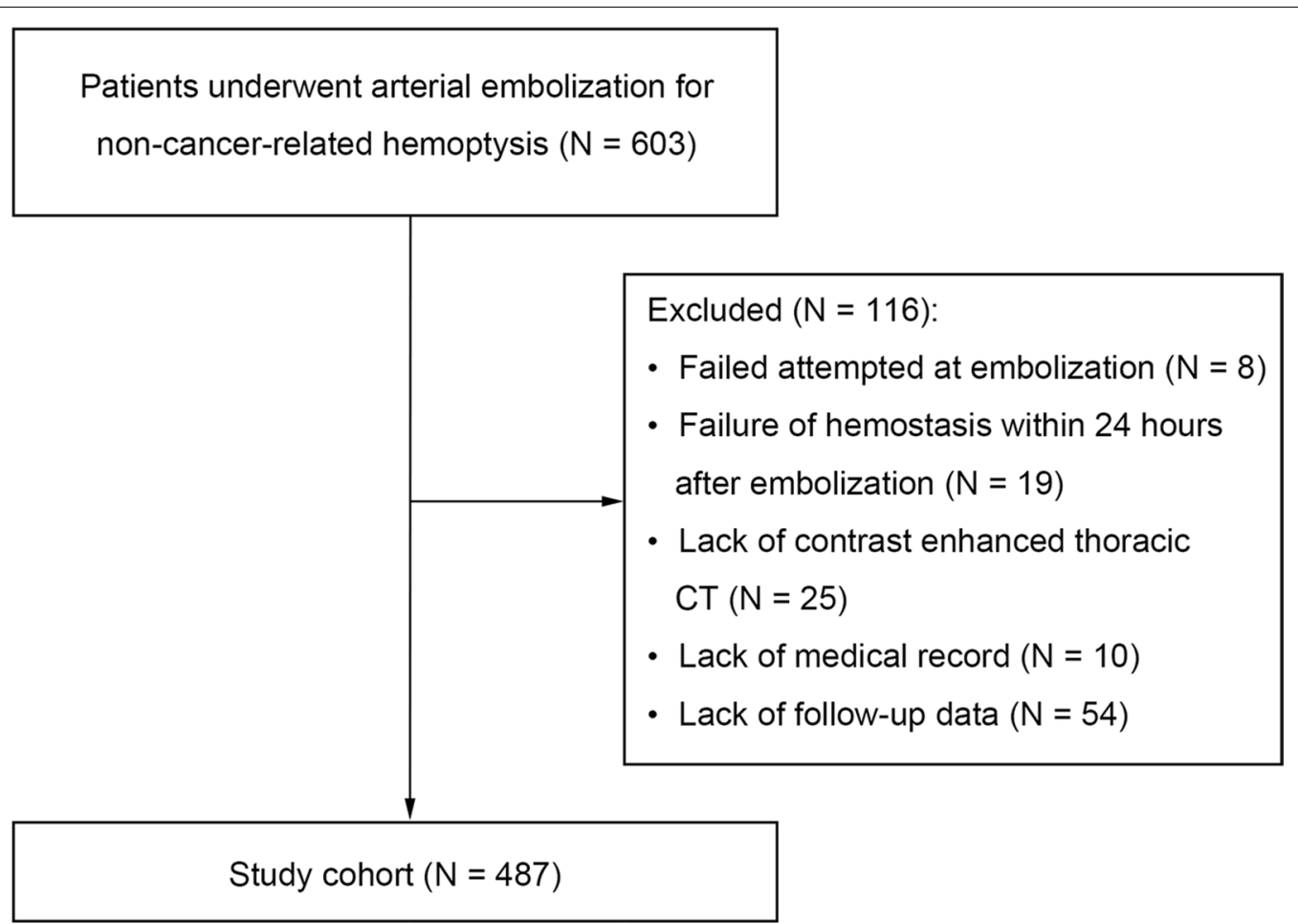

Fig. 1 Flowchart of enrolled patients 
recurrence, including patient demographics, clinical features, radiographic findings, and embolization procedure, were gathered. A subgroup of the study cohort, patients were diagnosed as bronchiectasis $(n=251)$, was analyzed and published in the Journal of CardioVascular and Interventional Radiology.

Hemoptysis severity were graded according to blood loss within $24 \mathrm{~h}$ as mild $(<100 \mathrm{ml})$, moderate (100$300 \mathrm{ml}$ ), and massive ( $\geq 300 \mathrm{ml}$ ) [2]. The CT angiography (CTA) was performed in our study with SOMATOM Force, Siemens, Germany; Revolution CT, GE Healthcare, United States; and Optima CT, GE Healthcare, United States with approximately $50-60 \mathrm{~mL}$ of contrast agent (Iodixanol $320 \mathrm{mgI} / \mathrm{mL}$, GE Healthcare or Iopromide $370 \mathrm{mgI} / \mathrm{mL}, \mathrm{BAYER})$, which was injected intravenously at a speed of $4.0 \mathrm{~mL} / \mathrm{s}$. Then all image data were transferred to a post-processing workstation and reconstructed at $1.25 \mathrm{~mm}$ section thickness. The culprit vessels on CTA were identified upon hypertrophy, hypervascularity, tortuosity, and aneurysmal. Patients were classified into three categories disease categories: bronchiectasis, tuberculosis (TB) sequela, and non-TB and non-bronchiectasis (chronic pneumonia, cryptogenic hemoptysis, aspergilloma and pneumoconiosis). Cryptogenic hemoptysis indicated the lack of specific lung parenchymal or vascular abnormality as noted on preoperative CTA [16]. The extent of pulmonary disease (number of affected lobes), presence of pleural thickening, and lung destruction were assessed. Pleura thickness $>3 \mathrm{~mm}$ was considered pathological [17]. Lung destruction was defined as diffuse lung parenchymal destruction or lung volume loss in at least one lobe on CT [18].

\section{Arterial embolization procedures}

Before procedure, interventional radiologists reviewed CTA to observe the lung lesion, culprit artery anatomy. We also searched suspicious non-bronchial systemic arteries (NBSAs) according to the location of lung lesion, including inferior phrenic arteries for lower lung lesion, internal mammary or esophageal arteries for medial lung lesion and superior thoracic arteries for upper lung lesion, etc. BAE was performed with Artis Zeego Digital Subtraction Angiography (DSA), Siemens, Germany; IGS5-4 DSA, GE, United States; UNIQ FD20 DSA, PHILIPS, Netherlands and UNIQ FD20/15 DSA, PHILIPS, Netherlands. All procedure was done via a transfemoral approach under local anesthesia. Angiography was performed by a $5 \mathrm{~F}$ catheter (Cobra, RLG, MIK catheter, Cook Medical, Bloomington, Indiana, USA) with a total amount of $6-10 \mathrm{~mL}$ contrast agent at a rate of $1.5-2.0 \mathrm{~mL} / \mathrm{s}$. Selective angiography of the bronchial arteries or suspicious NBSAs were recorded. Abnormal angiographic findings included abnormal culprit artery hypertrophy, distortion, neovascularity, hypervascularity, systemic arterial-pulmonary circulation shunts (SPS) or extravasation of the contrast agent [19]. Once the presence of abnormal vessels was confirmed, a microcatheter (2.7F; Terumo Medical Corp., Tokyo, Japan; or 2.4F; Merit Maestro, South Jordan, Utah, USA) was introduced and advanced to avoid the possible risk of non-target embolization. Embolization continued until complete occlusion of all bronchial and pathological NBSA was achieved. Embolization materials included polyvinyl alcohol (PVA) particles (300-500 $\mu \mathrm{m}$; Cook), microspheres (500-700 $\mu \mathrm{m}$; Merit Maestro), and gelatin sponge particles (350-560 $\mu \mathrm{m}$; Hangzhou Alicon Pharmaceutical Co., Ltd., Zhejiang, China). Micro-coils (COOK) were used in 25 patients after initial embolization with particles. Procedure-related complications were classified according to the Society of Interventional Radiology (SIR) guidelines [20].

\section{Follow-up and recurrence}

After discharge, patients were followed up by outpatient clinic during 1-3 months and then underwent telephone interview for the first two years. If no recurrence, followup cycle was extended to once a year. During the followup, healthy lifestyle education, antibiotics treatment and rehabilitation was encouraged for the better long-term outcome. Recurrence was defined as hemoptysis $\geq 30 \mathrm{ml}$ per day and needing further medical care, repeat BAE, lobectomy, or hemoptysis as a cause of death after clinical success $[11,21]$. The end date of follow-up was defined as date of death by any cause or May 2020. Recurrence-free time was the period from the date of embolization procedure to the date of recurrence, death, or last follow-up.

\section{Development and validation of the prognostic model}

We performed univariate and multivariate Cox regression analyses to determine recurrence risk factors from clinical and imaging signatures. Variables with significance in the univariable Cox regression analysis were included in the multivariate Cox analysis with backward selection method. The candidate clinical variables were age, sex, underlying lung disease, amount of hemoptysis, history of hemoptysis, smoking, hypertension, and previous lobectomy. Imaging features included the extent of pulmonary disease (number of affected lobes), presence of pleural thickening, lung destruction, number of culprit bronchial arteries, SPS, presence of culprit NBSAs, and type of embolization material.

Based on the results of multivariate regression, the risk prediction model for recurrence was formulated and presented with a nomogram. The regression coefficient of variables derived from multivariate Cox regression analysis was proportionally converted to a 0 - to 100 -point 
scale and each variable was listed separately. The points of the independent variables were added to derive total points, which was matched to a scale of outcome. The performance of the model was measured by the area under the receiver operating characteristic (ROC) curve (AUC) value, calibration curves, and Brier score.

\section{Statistical analysis}

Baseline data were expressed as mean (SD) or median (range) for quantitative variables and frequencies (percentage) for categorical variables. We used KaplanMeier analysis to estimate cumulative recurrence-free curves. Univariate and multivariate Cox regression analyses were applied to identify the independent predictors of recurrent hemoptysis. Bootstrapping with 1000 samples was used for model calibration validation. The discrimination performance of our model was quantified by the AUC value. The "Boot632plus" split method with 1000 iterations was used to calculate the prediction error of the current model. Estimates of prediction error were summarized as the integrated Brier score, which meant a weighted average of the squared distances between predicted probabilities and observed outcomes. The Brier score can reflect discrimination and calibration simultaneously; it can evaluate the overall model performance effectively. When a model is perfect, the Brier score will approach 0.0 . For a model with a $50 \%$ incidence of the outcome, the Brier score will equal 0.25 [22]. All statistical analyses were conducted with software $(\mathrm{R}$, version 4.0.2). $P$-values below 0.05 were considered statistically significant.

\section{Results}

\section{Characteristics of the study cohorts}

Table 1 shows baseline characteristics of the study patients. Median duration of hemoptysis was 7 days (range, 0 day-35 years). The common underlying lung diseases were bronchiectasis $(251 / 487,51.5 \%)$ and TB sequela (139/487, 28.5\%). In total, 1279 arteries including 1047 bronchial arteries (569 in right, 478 in left) and 232 NBSAs, were embolized.

\section{Complication and recurrence}

Minor complications occurred in 80 patients, including fever in 36 , chest or shoulder discomfort in 30 , abdominal discomfort or vomiting in 10, puncture site hematoma in two, headache in one, and anaphylaxis in one patient. One patient experienced a major complication. He suffered left extremity weakness one day after procedure and CT images showed cerebral lacunar infarction. This patient presented with bronchial artery-pulmonary artery shunt and was embolized
Table 1 Baseline characteristics of the study patients $(N=487)$

\begin{tabular}{|c|c|}
\hline Variables & Mean \pm SD/n (\%) \\
\hline Age (years) & $60.2 \pm 13.5$ \\
\hline Sex (female/male) & $139(28.5 \%) / 348(71.5 \%)$ \\
\hline Median duration of hemoptysis (range, d) & $6(0-12,775)$ \\
\hline \multicolumn{2}{|l|}{ Hemoptysis amount (ml/d) } \\
\hline$<100$ & $189(38.8 \%)$ \\
\hline $100-300$ & $212(43.5 \%)$ \\
\hline$\geq 300$ & $86(17.7 \%)$ \\
\hline \multicolumn{2}{|l|}{ Underlying lung disease } \\
\hline Bronchiectasis & $251(51.5 \%)$ \\
\hline TB sequela & $139(28.5 \%)$ \\
\hline Non-TB and non-bronchiectasis" & $97(19.9 \%)$ \\
\hline History of lobectomy & $12(2.5 \%)$ \\
\hline Smoking & $145(29.8 \%)$ \\
\hline Hypertension & $141(29.0 \%)$ \\
\hline Disease extent (number of affected lobes) & $2.4 \pm 1.0$ \\
\hline Presence of pleural thickening & $270(55.4 \%)$ \\
\hline Lung destruction & $29(6.0 \%)$ \\
\hline Number of culprit bronchial arteries & $2.2 \pm 1.0$ \\
\hline Presence of culprit NBSAs & $134(27.5 \%)$ \\
\hline Systemic arterial-pulmonary circulation shunts & $173(35.5 \%)$ \\
\hline \multicolumn{2}{|l|}{ Embolization materials } \\
\hline PVA particle & $394(80.9 \%)$ \\
\hline Microsphere & $70(14.4 \%)$ \\
\hline Gelatin sponge & $23(4.7 \%)$ \\
\hline Median follow-up duration (range, d) & $675(143-1943)$ \\
\hline Recurrence & 93 (19.1\%) \\
\hline
\end{tabular}

NBSAs, non-bronchial systemic arteries; PVA, polyvinyl alcohol; SD, standard deviation; TB, tuberculosis

"Non-TB and non-bronchiectasis included chronic pneumonia $(n=55)$, cryptogenic hemoptysis $(n=32)$, aspergilloma $(n=1)$ and pneumoconiosis $(\mathrm{n}=9)$

with 300-500 $\mu \mathrm{m}$ PVA particles. The modified Rankin Scores were 2 at discharge and remained stable at the three years' follow-up visit.

The median follow-up time was 675 days (range, 1431943 days). Recurrent hemoptysis events occurred in $93(19.10 \%)$ cases. Eighty (86.0\%) of the recurrent cases occurred within 2 years after BAE. The one-month, and 1-, 2-, 3- and 5-year cumulative recurrence-free rates of patients were $94.5 \%, 88.0 \%, 81.4 \%, 76.2 \%$ and $73.8 \%$, respectively. Among all patients with recurrence events, 47 (50.5\%) patients underwent repeated BAE, one (1.1\%) underwent a lobectomy, seven (7.5\%) died due to recurrence and 38 (40.9\%) received conservative management. Repeat BAE showed that causes of recurrent hemoptysis included missed culprit arteries $(n=11,23.4 \%)$, recanalization $(n=20,42.6 \%)$ and revascularization of the collateral circulation $(n=16$, $34.0 \%)$. 


\section{Development and validation of the prognostic model}

Univariate analysis showed that sex, underlying lung diseases, history of hemoptysis, history of lobectomy, presence of pleural thickening, lung destruction, the extent of pulmonary disease (number of affected lobes), SPS, presence of culprit NBSAs and type of embolization material were statistically significant factors affecting recurrence.
Multivariate analysis revealed that underlying lung diseases $(P=0.026)$ and SPS $(P<0.001)$ were most significant of recurrence (Table 2). Recurrence-free curves for patients classified by underlying lung diseases and the presence of SPS are shown in Fig. 2. Based on these findings, a nomogram for individual hemoptysis patient risk stratification was created (Fig. 3). The 1-year, 2-year,

Table 2 Univariate and multivariate analysis of the variables associated with recurrence of hemoptysis in patients after BAE treatment

\begin{tabular}{|c|c|c|c|c|c|c|}
\hline \multirow[t]{2}{*}{ Variables } & \multirow[t]{2}{*}{ Level } & \multirow[t]{2}{*}{$\mathbf{N}$} & \multicolumn{2}{|l|}{ Univariate } & \multicolumn{2}{|l|}{ Multivariate } \\
\hline & & & $\mathrm{HR}(95 \% \mathrm{Cl})$ & $P$ value & $\mathrm{HR}(95 \% \mathrm{CI})$ & $P$ value \\
\hline Age & - & 487 & $1.01(0.99-1.03)$ & 0.299 & & \\
\hline \multirow[t]{3}{*}{ Sex } & & & & 0.048 & & \\
\hline & Male & 348 & $0.65(0.43-1.00)$ & & & \\
\hline & Female & 139 & Reference & & & \\
\hline \multirow[t]{3}{*}{ The history of hemoptysis } & & & & $<0.001$ & & \\
\hline & $>6$ months & 163 & $2.47(1.64-3.71)$ & & & \\
\hline & $\leq 6$ months & 324 & Reference & & & \\
\hline \multirow[t]{4}{*}{ Hemoptysis amount } & & & & 0.771 & & \\
\hline & $\geq 300 \mathrm{ml} / \mathrm{d}$ & 86 & $1.21(0.68-2.16)$ & 0.508 & & \\
\hline & $100-300 \mathrm{ml} / \mathrm{d}$ & 212 & $1.14(0.72-1.79)$ & 0.583 & & \\
\hline & $<100 \mathrm{ml} / \mathrm{d}$ & 189 & Reference & & & \\
\hline \multirow[t]{4}{*}{ Underlying lung diseases } & & & & 0.001 & & 0.026 \\
\hline & TB sequela & 139 & $3.32(1.61-6.85)$ & 0.001 & $2.32(1.15-5.19)$ & 0.027 \\
\hline & Bronchiectasis & 251 & $1.84(0.90-3.77)$ & 0.096 & $1.43(0.72-3.16)$ & 0.338 \\
\hline & Non-TB and non-bronchiectasis & 97 & Reference & & Reference & \\
\hline \multirow[t]{3}{*}{ History of lobectomy } & & & & 0.015 & & \\
\hline & Present & 12 & $3.07(1.24-7.60)$ & & & \\
\hline & Absent & 475 & Reference & & & \\
\hline \multirow[t]{3}{*}{ Smoking } & & & & 0.298 & & \\
\hline & Present & 145 & $0.78(0.49-1.25)$ & & & \\
\hline & Absent & 342 & Reference & & & \\
\hline \multirow[t]{3}{*}{ Hypertension } & & & & 0.240 & & \\
\hline & Present & 141 & $0.75(0.47-1.21)$ & & & \\
\hline & Absent & 346 & Reference & & & \\
\hline Disease extent (number of affected lobes) & - & 487 & $1.42(1.18-1.72)$ & $<0.001$ & & \\
\hline \multirow[t]{3}{*}{ Presence of pleural thickening } & & & & $<0.001$ & & \\
\hline & Present & 270 & $2.39(1.52-3.76)$ & & & \\
\hline & Absent & 217 & Reference & & & \\
\hline \multirow[t]{3}{*}{ Lung destruction } & & & & $<0.001$ & & \\
\hline & Present & 29 & $4.20(2.44-7.20)$ & & & \\
\hline & Absent & 458 & Reference & & & \\
\hline Number of culprit BAs & - & 487 & $0.87(0.70-1.08)$ & 0.212 & & \\
\hline \multirow[t]{3}{*}{ Presence of culprit NBSAs } & & & & 0.004 & & \\
\hline & Present & 134 & $1.84(1.21-2.78)$ & & & \\
\hline & Absent & 353 & Reference & & & \\
\hline \multirow[t]{3}{*}{ Systemic arterial-pulmonary circulation shunts } & & & & $<0.001$ & & $<0.001$ \\
\hline & Present & 173 & $2.86(1.88-4.34)$ & & $2.49(1.63-3.87)$ & \\
\hline & Absent & 314 & Reference & & Reference & \\
\hline \multirow[t]{3}{*}{ Embolization materials } & & & & 0.004 & & \\
\hline & Gelatin sponge & 23 & $2.74(1.38-5.46)$ & & & \\
\hline & PVA particle and microsphere & 464 & Reference & & & \\
\hline
\end{tabular}

BA, bronchial arteries; BAE, bronchial arterial embolization; Cl, confidence interval; HR, hazard ratio; NBSAs, non-bronchial systemic arteries; PVA, polyvinyl alcohol; TB, tuberculosis; SD, standard deviation 

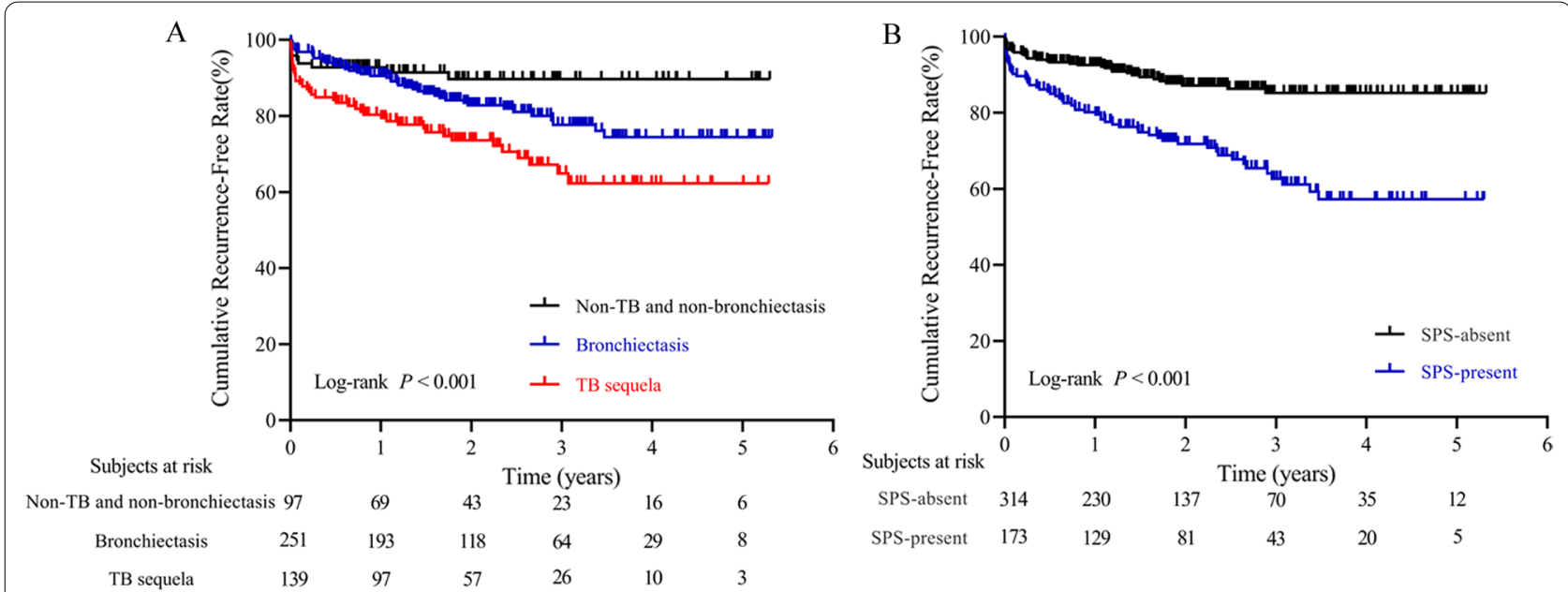

Fig. 2 Kaplan-Meier curves for recurrence-free time in all patients based on underlying lung diseases (A) and presence of systemic arterial-pulmonary circulation shunts (B)

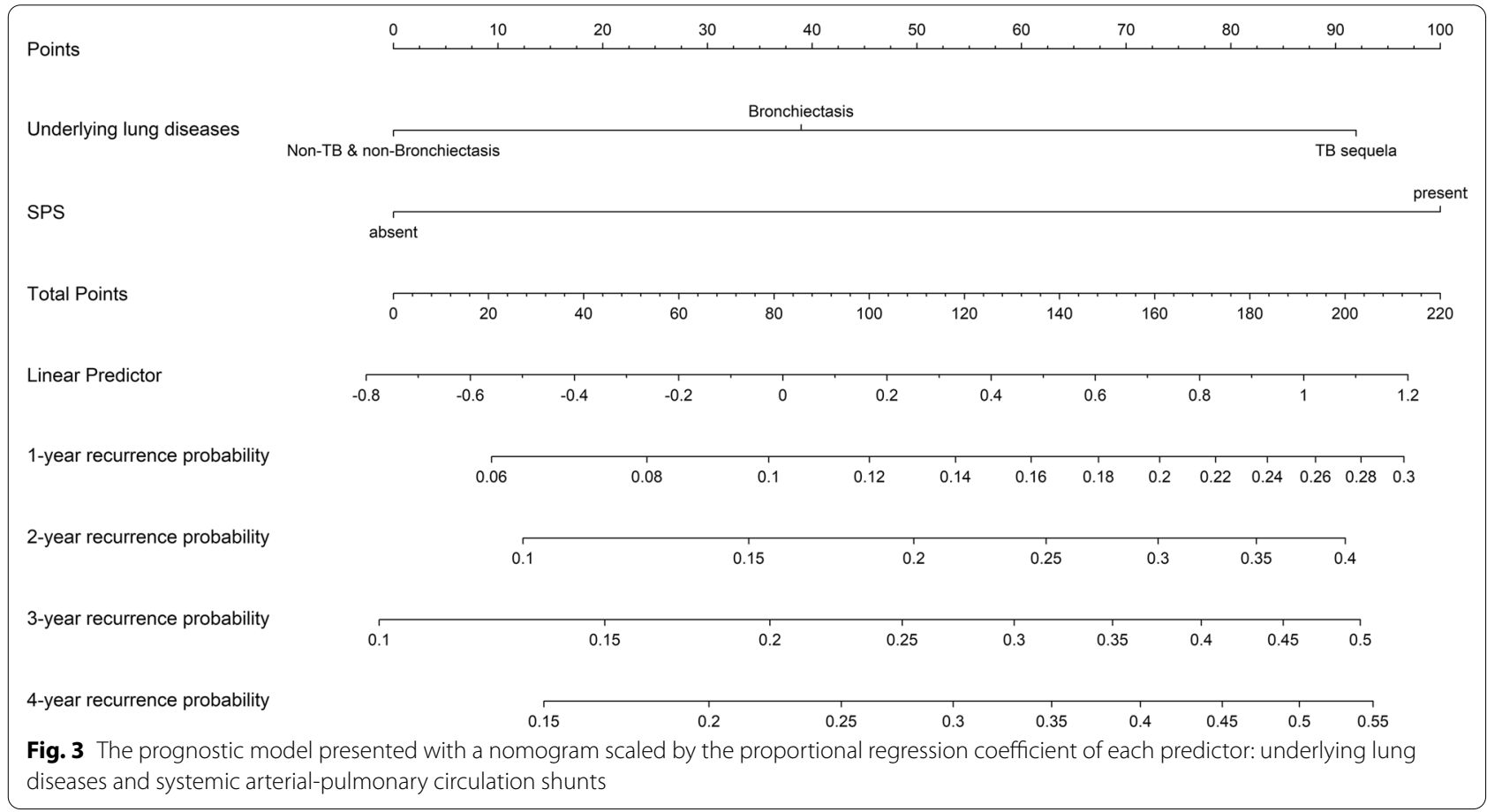

3-year and 4-year recurrence rates of individual patients could then be predicted after embolization. The model maintained good discriminatory performance (AUC, 0.69; 95\% CI 0.62-0.76). The time-dependent AUC value is shown in Fig. 4. The prediction error of our model was low (integrated Brier score, 0.143). The prediction error rate of recurrence at 4 years was about 20\% (Fig. 5). The calibration chart between predicted and observed recurrence probability was plotted. The accuracy of prediction for first 2-year recurrence was acceptable, while late recurrence prediction ability declined moderately (Fig. 6A-D).

\section{Discussion}

Results from our study showed BAE was highly effective in controlling hemoptysis, with 2-year and 5-year recurrence-free rates of $81.4 \%$ and $73.8 \%$, respectively. However, the cumulative recurrent rates did not improve 


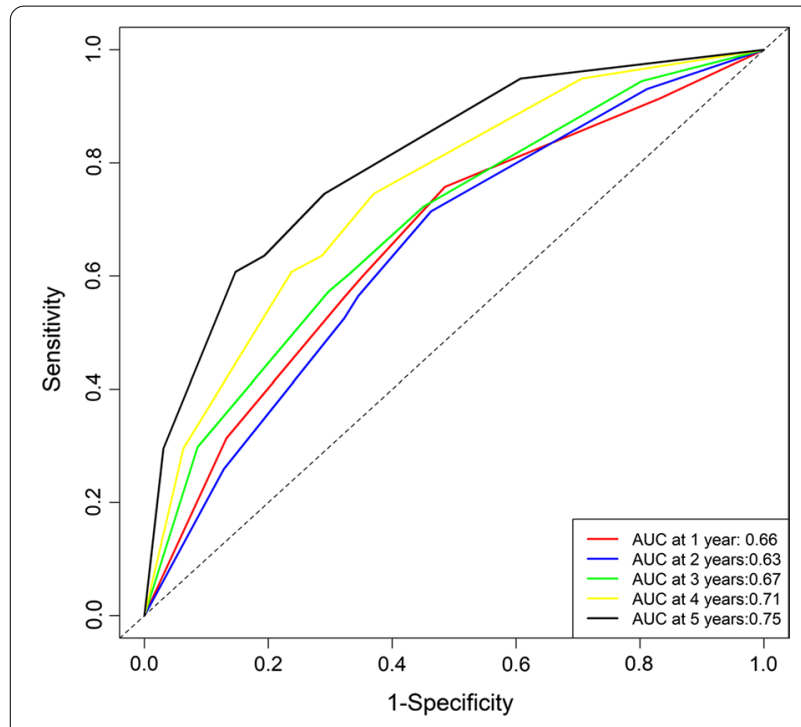

Fig. 4 The time-dependent ROC curve analysis for nomogram for patient cohorts

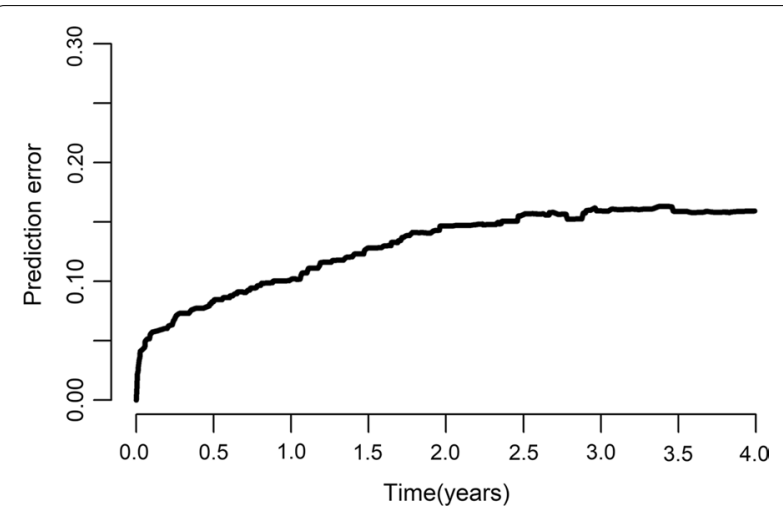

Fig. 5 The time-dependent prediction error rate for the model

significantly over a decade [2]. Based on the clinical and imaging parameters in this multi-institutional study, the prediction model accounted for the interaction of relevant and statistically significant factors to predict the recurrence of non-cancer-related hemoptysis in patients after embolization. Two factors, underlying lung diseases and SPS, were found most useful at predicting recurrence and incorporated into the model. The objectiveness and accessibility properties of these factors suggest that clinical application of the model will be straightforward. Results showed the model possessed good prognostic ability (AUC, 0.69; 95\% CI 0.62-0.76) and a lower prediction error (integrated Brier score, 0.143).

We enrolled non-cancer related hemoptysis patients with technical and clinical successful embolization procedures in this study to develop the model. The 1-, 2-, 3- and 5-year cumulative recurrence-free rates were $88.0 \%, 81.4 \%, 76.2 \%$ and $73.8 \%$. This was a promising long recurrence-free interval and could be explained by the differences in enrolled population, adequate preoperative CTA evaluation, reasonable antibiotic therapy and relative regular follow-up. During the median follow-up time of 675 days, 80 patients $(80 / 93,86.0 \%)$ experienced relapse within 2 years, while few recurrences occurred later. This 2-year recurrent peak period was also noted by others $[9,10]$. Taking this into consideration, patients should be closely followed-up for at least 2 years after the embolization. Calibration performance showed the model had a higher predictive power in the first 2 years' events. This inadequacy of the model to predict recurrence beyond 2 years after BAE may be explained by the nature of the underlying lung diseases. TB sequela and bronchiectasis with chronic inflammatory features accounted for $28.5 \%$ and $51.5 \%$ of our study population, respectively. During this 2-year period, arteriogenesis was likely driven by inflammatory mediators [23]. While embolization initially achieved stasis, promoting recovery and stabilization following embolization, long-term outcome of individuals of those respiratory inflammatory disorders would inevitably be affected by unquantifiable factors, such as climate, daily habits and comorbidities.

Pre-procedure CT and culprit vessels embolization reduced the post-BAE relapse [24]. After successfully controlling hemoptysis, our model showed the accurate prediction of the probability of relapse was independent of the underlying lung diseases. In chronic pathophysiological condition, proangiogenic factors, such as vascular endothelial growth factor and angiopoietin-1, were secreted, which promoted the development of new fragile bronchial vasculature and the remodeling of existing vessels $[25,26]$. Consistent with previous studies [9, $12,13]$, TB sequela was more commonly associated with recurrence. These findings argue for aggressive management of primary lung diseases to reduce hemoptysis recurrence. Therefore, we constructed a subgroup analysis focusing on idiopathic bronchiectasis to remove the influence of the underlying lung diseases and provide the corresponding treatment for this specific benign disease. And the results showed that the nomogram based on the idiopathic bronchiectasis patients maintained favorable predictability for recurrence and improved long-term outcomes of patients at risk of recurrence are expected with thorough follow-up, health education, and pulmonary rehabilitation [27]. As previous reported, SPS, as a specific angiographic finding during procedure, was again demonstrated to be an independent risk factor for recurrence after $\operatorname{BAE}[3,4,10,14]$. These two factors 

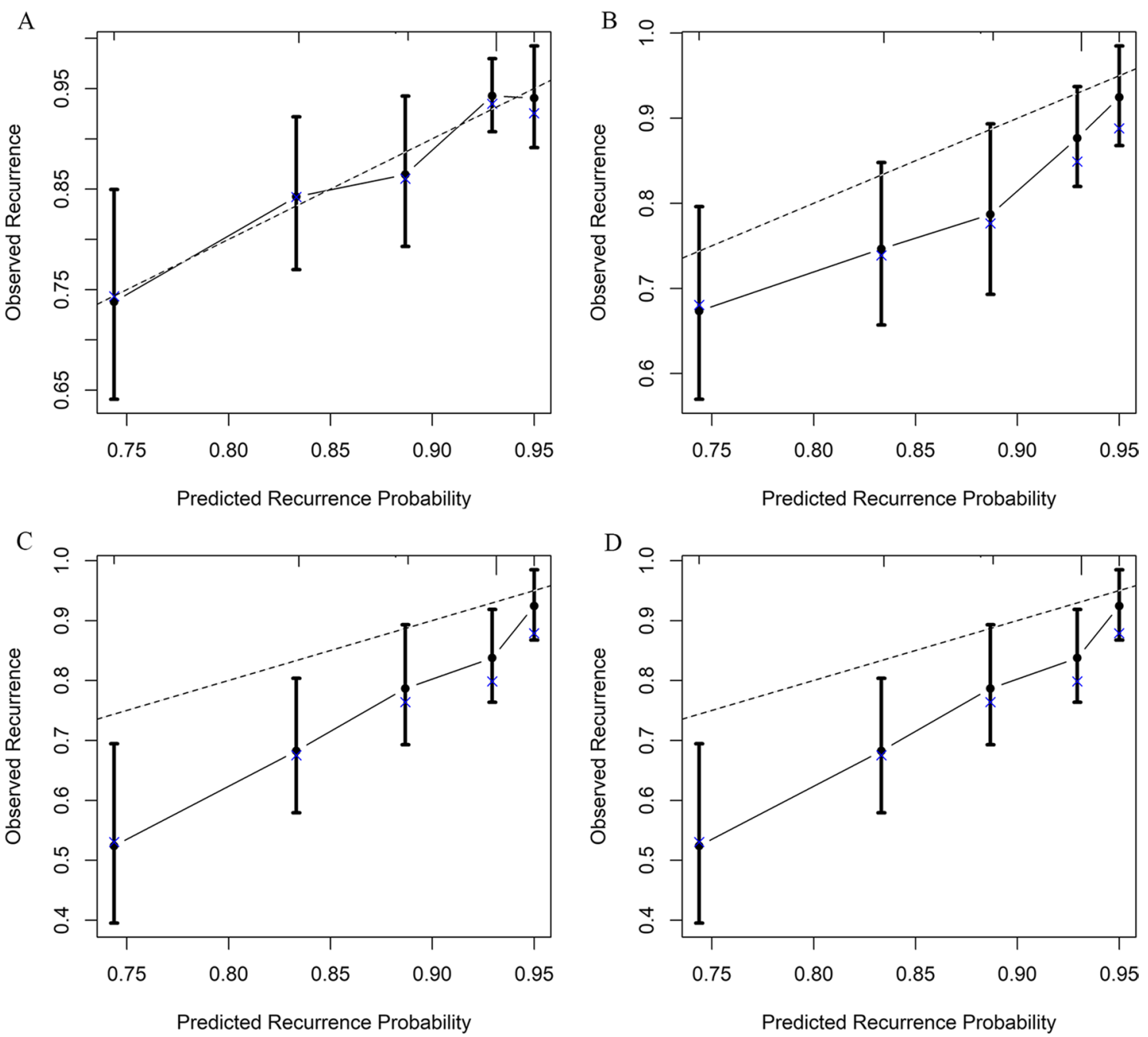

Fig. 6 A-D The time-dependent calibration curve of the model at 1-,2-, 3-, and 4-year after BAE

encompassed clinical and imaging parameters, and as part of our model, resulted in good discriminatory prediction and applicability. However, the existence of SPS and its impact on recurrence is not uniformly acknowledged $[4,9,10,28]$. Normally, the systemic and pulmonary circulations communicate at the capillary and precapillary levels [29]. If the pulmonary circulation was compromised, impairment of the pulmonary circulation and an increased demand for oxygen in local lung tissue happened. Systemic arteries supplying the lung, mainly bronchial arteries, often increase their flow to compensate for decreased lung perfusion [30, 31]. However, under such scenarios, the communications between the systemic arteries and pulmonary micro-circulation are prone to bleeding [32]. In general, BAE in individuals with SPS was safe. However, one patient with a shunt suffered left extremity weakness one day after procedure and CT images showed multi-lacunar infarction. We inferred that it might be related with BAE procedure, but not sure. The safety and influence of shunts during BAE warrants further study.

Certain limitations of this study should be pointed out. First, the study was retrospective and is predicted to result in bias. To minimize the bias of post-hoc analysis, an internal validation was performed. Ultimately, prospective validation is needed to verify the predictive performance of this model. Second, the primary disease spectrum in our cohort differed from that in previous studies. For example, aspergilloma was reported to be associated with hemoptysis recurrence [9, 33]. However, we could not analyze this risk factor because only one patient in our study was diagnosed with aspergilloma and did not experience relapse. Third, laboratory results were not analyzed, due to their high variability 
in the context of long-term events prediction. Fourth, the model did not assess the effects of medical treatment for pulmonary diseases that were coincident with or initiated after BAE.

In conclusion, a model incorporating underlying lung diseases and SPS demonstrated good performance for predicting the recurrence of non-cancer-related hemoptysis after embolization. The model may assist clinicians in identifying higher-risk patients to improve the longterm efficacy of BAE.

\section{Abbreviations}

BAE: Bronchial arterial embolization; Cl: Confidence interval; CTA: CT angiography; DSA: Digital Subtraction Angiography; HR: Hazard ratio; NBSAs: Nonbronchial systemic arteries; PVA: Polyvinyl alcohol; ROC: Receiver operating characteristic curve; SIR: Society of interventional radiology; SPS: Systemic arterial-pulmonary circulation shunts; TB: Tuberculosis.
\end{abstract}

\section{Authors' contributions}

Concepts/study design: H-TY, G-DL, JL, H-BS, Q-QZ; data analysis/ interpretation: H-TY, G-DL, J-XZ, JL, Q-QZ; data collection: H-TY, G-DL, X-ZH, D-ZZ, K-YG, J-XZ; manuscript drafting: H-TY, G-DL, JL, SL, Q-QZ; critical revision of the article: JL, SL, H-BS, Q-QZ; final approval of the article: all authors; statistical analysis: JL; overall responsibility: H-BS, Q-QZ. All authors read and approved the final manuscript.

\section{Funding}

This study was funded by Jiangsu Province's Key Talents Program (QNRC2016559 to Qing-Quan Zu) and Construction Program of Jiangsu Province Clinical Research Center Support System (BL2014084 to Qing-Quan Zu). We thank Prof. Frederick S. Keller, M.D., and Prof. Khashayar Farsad, MD, PhD, Charles T. Dotter Department of Interventional Radiology, Oregon Health \& Sciences University for their linguistic assistance and scientific consultation during the preparation of this manuscript.

\section{Availability of data and materials}

The data that support the findings of this study are available from the corresponding author upon reasonable request.

\section{Declarations}

\section{Ethics approval and consent to participate}

Study protocol followed the guidelines of the World Medical Association Declaration of Helsinki and were approved by the Ethics Committee of the First Affiliated Hospital with Nanjing Medical University (Ethical review no. 2018-SR097). Written informed consent was not required for this retrospective study.

\section{Consent for publication}

Not applicable.

\section{Competing interests}

The authors declare that they have no competing interests.

\section{Author details}

'Department of Interventional Radiology, The First Affiliated Hospital With Nanjing Medical University, No. 300 Guangzhou Road, Nanjing 210029, China. ${ }^{2}$ Department of Interventional Radiology, Jiangyin People's Hospital, Wuxi 214400, China. ${ }^{3}$ Department of Interventional Radiology, Jiangsu Taizhou People's Hospital, Taizhou 225300, China. ${ }^{4}$ Department of Interventional Radiology, Yixing People's Hospital, Wuxi 214200, China. ${ }^{5}$ Department of Clinical Medicine Research Institution, The First Affiliated Hospital with Nanjing Medical University, Nanjing 210029, China.

Received: 14 July 2021 Accepted: 6 December 2021

Published online: 18 December 2021

\section{References}

1. Mondoni M, Carlucci P, Job S, et al. Observational, Multicentre study on the epidemiology of haemoptysis. Eur Respir J. 2018;51:1701813. https:// doi.org/10.1183/13993003.01813-2017.

2. Panda A, Bhalla AS, Goyal A. Bronchial artery embolization in hemoptysis: a systematic review. Diagn Interv Radiol. 2017;23:307-17. https://doi.org/ 10.5152/dir.2017.16454

3. Chen J, Chen LA, Liang ZX, et al. Immediate and long-term results of bronchial artery embolization for hemoptysis due to benign versus malignant pulmonary diseases. Am J Med Sci. 2014;348:204-9. https:// doi.org/10.1097/MAJ.0000000000000226.

4. Lu GD, Zu QQ, Zhang JX, et al. Risk factors contributing to early and late recurrence of haemoptysis after bronchial artery embolisation. Int J Tuberc Lung Dis. 2018;22:230-5. https://doi.org/10.5588/ijtld.17.0543.

5. Kato A, Kudo S, Matsumoto K, et al. Bronchial artery embolization for hemoptysis due to benign diseases: immediate and long-term results. Cardiovasc Intervent Radiol. 2000;23:351-7. https://doi.org/10.1007/ s002700010062.

6. Syha R, Benz T, Hetzel J, et al. Bronchial artery embolization in hemoptysis: 10-year survival and recurrence-free survival in benign and malignant etiologies: a retrospective study. Rofo. 2016;188:1061-6. https://doi.org/10.1055/s-0042-112227.

7. Woo S, Yoon CJ, Chung JW, et al. Bronchial artery embolization to control hemoptysis: comparison of $n$-butyl-2-cyanoacrylate and polyvinyl alcohol particles. Radiology. 2013;269:594-602. https://doi.org/10. 1148/radiol.13130046.

8. Garcia-Olive I, Sanz-Santos J, Centeno C, et al. Predictors of recanalization in patients with life-threatening hemoptysis requiring artery embolization. Arch Bronconeumol. 2014;50:51-6. https://doi.org/10. 1016/j.arbres.2013.06.003.

9. Chun JY, Belli AM. Immediate and long-term outcomes of bronchial and non-bronchial systemic artery embolisation for the management of haemoptysis. Eur Radiol. 2010;20:558-65. https://doi.org/10.1007/ s00330-009-1591-3.

10. Choi J, Baik JH, Kim CH, et al. Long-term outcomes and prognostic factors in patients with mild hemoptysis. Am J Emerg Med. 2018;36:1 1605. https://doi.org/10.1016/j.ajem.2017.11.053.

11. van den Heuvel MM, Els Z, Koegelenberg CF, et al. Risk factors for recurrence of haemoptysis following bronchial artery embolisation for life-threatening haemoptysis. Int J Tuberc Lung Dis. 2007;1 1:909-14.

12. Shin $B S$, Jeon GS, Lee $S A$, et al. Bronchial artery embolisation for the management of haemoptysis in patients with pulmonary tuberculosis. Int J Tuberc Lung Dis. 2011;15:1093-8. https://doi.org/10.5588/ijtld.10. 0659.

13. Pei R, Zhou Y, Wang G, et al. Outcomes of bronchial artery embolization for life-threatening hemoptysis secondary to tuberculosis. PLOS ONE. 2014;9:e115956. https://doi.org/10.1371/journal.pone.0115956.

14. Xu S, Guan LJ, Shi BQ, et al. Recurrent hemoptysis after bronchial artery embolization: prediction using a nomogram and artificial neural network model. AJR Am J Roentgenol. 2020;215:1490-8. https://doi.org/ 10.2214/AJR.20.22775

15. Swanson KL, Johnson CM, Prakash UB, et al. Bronchial artery embolization: experience with 54 patients. Chest. 2002;121:789-95. https://doi. org/10.1378/chest.121.3.789.

16. Lee H, Yoon CJ, Seong NJ, et al. Cryptogenic hemoptysis: effectiveness of bronchial artery embolization using $n$-butyl cyanoacrylate. J Vasc Interv Radiol. 2017;28:1161-6. https://doi.org/10.1016/j.jvir.2017.03. 029.

17. Yoon W, Kim YH, Kim JK, et al. Massive hemoptysis: prediction of nonbronchial systemic arterial supply with chest CT. Radiology. 2003;227:232-8. https://doi.org/10.1148/radiol.2271020324.

18. Kim HC, Kim TH, Kim YJ, et al. Effect of tiotropium inhaler use on mortality in patients with tuberculous destroyed lung: based on linkage between hospital and nationwide health insurance claims data in South Korea. Respir Res. 2019;20:85. https://doi.org/10.1186/s12931-019-1055-5.

19. Davidson K, Shojaee S. Managing massive hemoptysis. Chest. 2020;157:77-88. https://doi.org/10.1016/j.chest.2019.07.012.

20. Angle JF, Siddiqi NH, Wallace MJ, et al. Quality improvement guidelines for percutaneous transcatheter embolization: society of interventional radiology standards of practice committee. J Vasc Interv Radiol. 2010;21:1479-86. https://doi.org/10.1016/j.jvir.2010.06.014. 
21. Anuradha C, Shyamkumar NK, Vinu M, Babu NR, Christopher DJ. Outcomes of bronchial artery embolization for life-threatening hemoptysis due to tuberculosis and post-tuberculosis sequelae. Diagn Interv Radiol. 2012;18:96-101. https://doi.org/10.4261/1305-3825.DIR.3876-11.2.

22. Cohen ME, Ko CY, Bilimoria KY, et al. Optimizing ACS NSQIP modeling for evaluation of surgical quality and risk: patient risk adjustment, procedure mix adjustment, shrinkage adjustment, and surgical focus. J Am Coll Surg. 2013;217:336-46. https://doi.org/10.1016/j.jamcollsurg.2013.02.027.

23. Hsia CC. Comparative analysis of the mechanical signals in lung development and compensatory growth. Cell Tissue Res. 2017;367:687-705. https://doi.org/10.1007/s00441-016-2558-8.

24. Li PJ, Yu H, Wang Y, et al. Multidetector computed tomography angiography prior to bronchial artery embolization helps detect culprit ectopic bronchial arteries and non-bronchial systemic arteries originating from subclavian and internal mammary arteries and improve hemoptysis-free early survival rate in patients with hemoptysis. Eur Radiol. 2019;29:19508. https://doi.org/10.1007/s00330-018-5767-6.

25. McDonald DM. Angiogenesis and remodeling of airway vasculature in chronic inflammation. Am J Respir Crit Care Med. 2001;164:S39-45. https://doi.org/10.1164/ajrccm.164.supplement_2.2106065.

26. Larici AR, Franchi P, Occhipinti $M$, et al. Diagnosis and management of hemoptysis. Diagn Interv Radiol. 2014;20:299-309. https://doi.org/10. 5152/dir.2014.13426.

27. Yan HT, Lu GD, Huang XZ, et al. A nomogram to predict recurrence after bronchial artery embolization for hemoptysis due to bronchiectasis. Cardiovasc Intervent Radiol. 2021;44:1609-17. https://doi.org/10.1007/ s00270-021-02923-0.

28. Lu GD, Zhang JX, Zhou CG, et al. Arterial embolization for hemoptysis in patients with chronic pulmonary tuberculosis and in patients with bronchiectasis. Acta Radiol. 2019;60:866-72. https://doi.org/10.1177/02841 85118805258

29. Yon JR, Ravenel JG. Congenital bronchial artery-pulmonary artery fistula in an adult. J Comput Assist Tomogr. 2010;34:418-20. https://doi.org/10. 1097/RCT.0b013e3181d1e96e.

30. Almeida J, Leal C, Figueiredo L. Evaluation of the Bronchial arteries: normal findings, hypertrophy and embolization in patients. Insights Imaging. 2020;1 1:70. https://doi.org/10.1186/s13244-020-00877-4.

31. Walker CM, Rosado-de-Christenson ML, Martinez-Jimenez S, et al. Bronchial Arteries: anatomy, function, hypertrophy, and anomalies. Radiographics. 2015;35:32-49. https://doi.org/10.1148/rg.351140089.

32. VanDerPloeg DG, Strong WR, Krohmer SJ, et al. Congenital bronchial artery to pulmonary artery fistula presenting as hemoptysis. Ann Thorac Surg. 2015;99:e19-20. https://doi.org/10.1016/j.athoracsur.2014.10.057.

33. Le HY, Le VN, Pham NH, et al. Value of multidetector computed tomography angiography before bronchial artery embolization in hemoptysis management and early recurrence prediction: a prospective study. BMC Pulm Med. 2020;20:231. https://doi.org/10.1186/s12890-020-01271-y.

\section{Publisher's Note}

Springer Nature remains neutral with regard to jurisdictional claims in published maps and institutional affiliations.

Ready to submit your research? Choose BMC and benefit from:

- fast, convenient online submission

- thorough peer review by experienced researchers in your field

- rapid publication on acceptance

- support for research data, including large and complex data types

- gold Open Access which fosters wider collaboration and increased citations

- maximum visibility for your research: over 100M website views per year

At BMC, research is always in progress.

Learn more biomedcentral.com/submissions 